\title{
Demographic assessment and adjustment of census age distribution in selected West African Countries
}

\author{
Elizabeth O. Njoku' ${ }^{1}$, David O. Okunlola ${ }^{1}$ \& Sunday A. Adedini ${ }^{1,2}$ \\ ${ }^{1}$ Demography and Social Statistics Department, Obafemi Awolowo University, \\ lle-Ife, Nigeria \\ ${ }^{2}$ Programme in Demography and Population Studies, Schools of Public Health \\ and Social Sciences, University of the Witwatersrand, \\ Johannesburg, South Africa \\ E-mail: hielizabethogechi@gmail.com
}

\begin{abstract}
Context/background: Despite the public acknowledgment of improvement in sub-Saharan African censuses and surveys in the recent time, the quality of age-sex data still remains relatively poor. This study assessed the quality of reported age-sex data in selected West African countries.

Data source and methods: We utilized the available recent West African census data to address the study objective. Data adjustments were made using the UN-moving average technique, Carrier Far-rag, Karup-king newton and Arriaga methods.

Results: First, the age- distribution in five years was examined for errors. The UN-Age-sex accuracy index showed that all the selected countries had inaccurate age-distribution, with the exception of Cameroon (2005) and Senegal (2002) censuses which revealed a lesser distorted age- distribution. Utilizing the strong smoothing techniques, the results showed that virtually all the censuses were plaqued with the overestimation error at older ages, particularly the female population. The error margin ranged between $10-25 \%$.

Conclusion: The study concludes that inaccuracies in West African census data persist and recommends more quality age-sex data in region.
\end{abstract}

Keywords: Age misstatement, Age distribution, Adjustment, West Africa

\section{Introduction}

Across all levels of governance, data classified by age has been increasingly used as a basis for socioeconomic, political, health and developmental planning, policy design and distribution of resources. Unfortunately for developing countries, their continuous quest to keep pace with the population policies aimed at reducing population growth and achieving overall development, have re-currently been thwarted due to inaccuracies in age data (Adebowale, Fagbamigbe, \& Bello, 2012; Mba, 2004, 2006, 2014; Randall \& Coast, 2016; Raymer \& Rogers, 2007). Censuses and surveys have been major sources of age data in the developing countries due to the difficulty in the availability and completeness of other sources of age data such as vital statistics, civil registrations and population register (Makannah, 1990; Sembajwe, 1990; Williams, 2014). West Africa, houses the most populous countries in sub-Saharan Africa especially in figures Nigeria as an example. Undoubtedly, the quantity and quality of the regions' age data raises reliability question as the basic error from censuses and survey have been linked to the technical and political context (Masquelier et al., 2016; Williams, 20I4) as well as the personnel's involved (i.e individuals and enumerators) (Adebowale et al., 2012; Gibril, 1979; Mba, 2004). Error-ridden data have implication for the fundamental estimation of demographic parameters (Adebowale et al., 20I2) and components of population dynamics (Coale \& Kisker, 1986).

While age misstatement persists and the data quality not ascertained (Chevan \& Sutherland, 2009; Gibril, 1979) the indices assessment techniques (Spoorenberg, 2007) like Myer's, Whipple's and Bachi's indexes have been employed in most recent multi-country African studies to assess the magnitude of digit preference in age data (Mba, 2003, 20I4; Randall \& Coast, 2016), no attempt have been made to refine the age data across all age sub-groups either utilizing the demographic techniques or to further estimate the population parameters as was done in the study of (Adebowale et al., 2012; Randall \& Coast, 2016) Although, the latter study utilized a multi-source, it was not a multi-country study. This identified gap necessitated this study in that available 
and accessible age data of the last two censuses of West African countries elicited from IPUMS was utilized in an attempt to assess and modify the age data of West Africa countries using the demographic techniques. In this way, contributing to the improvement of the quality of age distribution of the observed population which have developmental planning and policy implications.

\section{Literature review}

As far as 1960, a decennial census exercise had begun in some African countries and three census rounds (1990, 2000 and 2010) have been observed (UN, 2016). In all the 17 countries in West Africa, only Liberia missed out in the first two consecutive rounds, but Ghana, Cameroon, Chad, and Nigeria have missed one of the three rounds. Besides some underlining fundamental error in censuses which have been argued to also affect the national representative sample surveys like DHS (Williams, 20 I4), data from these sources have aided development in the region.

The continuous public demand and use of age as a requisite criterion in administrative issues in Africa have led to some variances in the levels and patterns of age distortions observed in the regions' censuses across all the population sub-groups (Mba, 2004, 20 I4; Randall \& Coast, 20 I6). This recurrent errors in age distribution have been majorly classified into coverage and content errors. The former relates to errors due to completeness, which is difficult in real life, as an individual could either be omitted (underenumeration) or counted more than once (overenumeration). Alternatively, the content error is tied to the whole process of the census. This includes respondent errors in reporting their ages which may be due to their ignorance or biases, educational level, preferences like sex selectivity in age preferences or avoidance (Makannah, 1990; Mba, 2003; Siegel, 2004). Likewise, enumerators biases or mistakes in recording age data, tabulating and coding errors (Siegel, 2004).

One of the recurrent trends and patterns of misreporting in some countries in West Africa is to under-report the age of children and that of adolescents, when they are far or near puberty or unmarried, and to over-report age when pubescent or married. For example, in Gambia, Senegal, Mali, Burkina-Faso and Ghana etc, the observed irregularities in the population pyramid around ages I 5 to 20 , is rooted in under-reports in age brackets from 10 to 24 years which in turn favor the two year age- groups of 5-9 and 25-29 (Ewbank, 198I; Gendreau \& Nadot, 1967; Hertrich \& Lardoux, 2014; Mba, 20/4). Also sex-selective age inflation is another observed trend for men i.e exaggeration of age by 3 years on average between 30 and 49 years of age, and at least 5 years beyond age 50 , while age avoidance and age inflation is the case for the women i.e high possibilities to report lower ages when below 40 years and overstated when above 40 years. Generally, enumerators in censuses and survey exercises may have challenges of age precision (Gilles Pison \& Ohadike, 2006), reporting ages ending in even numbers (Mba, 2004), exact ages when age heaping is either sensitive or preference driven i.e ages ending in 0 or 5 (Gendreau \& Nadot, 1967; Mba, 2004, 2006, 2014; Udjo, 2005) or avoiding reporting deficit in ages ending " 0 and 5" as in Guinea 1955 and surplus in others i.e in digits "4 or 9" as in Senegal census of I 988 (G Pison, Hill, Cohen, \& Foote, 1995). However, demographers and other population scientist such as (Axinn, Pearce, \& Ghimire, 1999) suggested the use of the life history calendars and landmark events ,as strategies to better elicit good age data , the procedures which was also approved by (Helleringer et al., 20|4).

Several approaches have been adopted for the errors and bias diagnosis and this includes Postenumeration surveys (PES) and matching of Independent surveys, comparison of sources, crosstabulating by age, cohort survival rates and stable population analysis. While the census survival analysis is also a very good measures in assessing age data and estimating the components of change especially when countries have a well-established practice of having census continuously overtime, PES dual-system estimation seemed to be the best analytical measure in that it provides the variances in content bias and also seemed best estimating coverage errors especially where data on the components of population change is reasonably in-accurate i.e migration, fertility and mortality rates, as in West Africa. This measure is also limited partly on the objectives of conducting PES as the probability of obtaining a better measure is dependent on accurate reporting of age in the PES as against the original census or survey and the cost of re-interview(Krótki, 1978). This method has been applied to study age misreporting in US and Ghana in 1960 and 1964 respectively (Ewbank, 198I). Recent studies have also analyzed age misreporting by comparing age data from different data sources in addition to the use of other analytical techniques like cross-tabulating approach commonly referred to as the indices techniques used in the study of (Randall \& Coast, 2016) or stable population analysis used in (Adebowale et al., 20।2). In these studies, whilst the target population, analytical approach, the study focus and scope differed, the results obtained from both measures had credits of satisfying the assumptions of the techniques used as well as the focus of the study. Although it appeared that the stable population 
analysis seems preferred over the indices analysis because of the advantage of either age error comparison from different sources or the refinement of age data as well. Also using indices analysis which also has also an added methodological advantage of doing the graphical analysis first to understand the distortions in the age distribution and to inform the choice of the type of indices analysis (Arriaga, Johnson, \& Jamison, 1994). The choice of Whipple's index in (Randall \& Coast, 2016) was beneficial in providing information on the individual tendencies to misreport age as well as the extent of systematic age heaping in ages ending with digits 0 and 5 but this method is also limited in that it is a fair measure rather than been a better estimates of the overall reliability of the age distributions (UN, 1986 ). Besides, that these two studies made a plausible impact having attempted to make some modifications to the methodologies utilized, they concluded that the countries reviewed had poor age data.

Considering the previous works of (Mba, 2004, 2006) unlike his more recent study (Mba, 20I4) which was more expansive in that he utilized multicountry censuses at different dates to evaluate age misreporting applying similar analytical technique as in (Randall \& Coast, 20/6), only that in the latter study the authors made some specific refinement of age data utilizing similar analytical technique as in (Mba, 20|4). These studies also concluded that the age data quality was poor but there were no attempts made to better refine the observed irregularities across all the reported ages, a gap this study intends to fill knowing the implications of such attempts on the age data quality so as to provide a relatively fair data for further estimation of population parameters which is useful to policy planners and decisions makers.

\section{Data and methods}

This study is focused on cross-country analysis which UNAI is most suitable in providing a summary index instead of the choice to use Whipple's, Bachi or Myres Indexes which are more of individual tendency measures (Hobbs, 2004; UN, 1955). Although the index does not consider real irregularities in age groups such as mortality caused by natural occurrences, human mortal activities, temporary fertility reduction, migratory movements among some age groups and sex, it, however, performs well in larger population which permits a neglect of any chance of irregularity or fluctuations.

Lastly, the demographic adjustment techniques such as the light smoothing techniques such as Carrier Farrag, United Nation Moving Average, Karup King Newton and Arriaga methods where were all employed and compared, hence the best performing method in assessing the extent and distribution of age-sex specific coverage errors was identified as taken as the assumed true population figure. The best method was assessed with the error percent. All these are based the assumptions of no or insignificance distortions in fertility, mortality and migration in the selected West African countries namely Burkina-Faso, Cameroun, Ghana, Mali, and Senegal, hence the applicability.

\section{(a) Average sex ratio score (S)}

This score is obtained by first calculating the sex ratio at each age group. Successive differences irrespective of the sign are added and averaged.

$$
\begin{aligned}
& \text { Age-specific sex ratio }=\frac{5 \mathrm{P}_{x}^{m}}{5 \mathrm{P}_{x}^{f}} \times 100 \\
& 5 \mathrm{P}_{x}^{m}=\text { males aged } \mathrm{x} \text { to } \mathrm{x}+5 \\
& 5 \mathrm{P}_{x}^{f}=\text { females aged } \mathrm{x} \text { to } \mathrm{x}+5
\end{aligned}
$$

\section{(b) Average male age ratio score (M)}

For each age group for males, calculate the age ratios computed as

$$
\text { Age ratio }=\frac{5 \mathrm{P}_{x}^{m}}{\frac{1}{2}\left(5 \mathrm{P}_{x-5}^{m-5}+5 \mathrm{P}_{x+5}^{m}\right)} \times 100
$$

The deviations from unity irrespective of the sign are added and averaged (M).

\section{(I) Sex ratio}

Sex ratio is the ratio of males to females in a given population, usually expressed as number of males for every 100 females i.e. $\frac{p_{i}^{m}}{p_{i}^{f}} \times 100, i=$ each 5 -year age group

\section{Carrier - Farrag graduation method}

This method consists essentially of splitting the total numbers reported in two adjacent five-year age groups (quinary age groups) by first grouping the quinary age groups into 10 -years age groups (denary age groups). The graduate population is then obtained by the Manipulation of simple algebraic formulae i.e.

$$
\begin{aligned}
& \mathrm{Di}=\text { individual } \mathrm{I} 0 \text {-year age group } \\
& \mathrm{K} 4=\frac{\mathrm{v}_{i-1}}{\mathrm{v}_{i-1}}, \quad \mathrm{~K}=\sqrt[4]{\frac{\mathrm{v}_{i-1}}{v_{i+1}}}
\end{aligned}
$$

Where $\mathrm{K}=\mathrm{a}$ constant which depicts the average rate

$$
\begin{aligned}
& V i=\frac{D_{i}}{1+K} \\
& \therefore \quad \text { Smoothed Population }(P)=D i-V i
\end{aligned}
$$

\section{Karup King Newton}

The Karup King Formulas (Carrier \& Farrag, 1959) are as follows:

$$
\begin{aligned}
& { }_{5} P_{x}=\frac{1}{2} \quad{ }_{10} P_{x}+\frac{1}{16}\left({ }_{10} P_{x-10}-10 P_{x-10}\right) \text { and } \\
& { }_{5} P_{x+5}={ }_{10} P_{x}-{ }_{5} P_{x}
\end{aligned}
$$




\section{Arriaga method}

This method does not modify the total i.e. it takes age data in 10 -year and redistributes for to get 5year age groups. Also, separate the first and last I0-year age groups, unlike the other methods.

$$
\begin{aligned}
& 5 \mathrm{P}_{X+5}= \\
& \left.10\left(\mathrm{P}_{X-10}\right)+11\left(10 \mathrm{P}_{\mathrm{x}}\right)+2\left(10 \mathrm{P}_{\mathrm{x}+10}\right)\right) \frac{1}{16} \\
& { }_{5} \mathrm{P}_{\mathrm{x}+5}=\left(-{ }_{10} \mathrm{P}_{\mathrm{x}-10}+1 \mathrm{I}_{10} \mathrm{P}_{\mathrm{x}}+2{ }_{10} \mathrm{P}_{\mathrm{x}+10}\right) \frac{1}{24} \\
& { }_{5} \mathrm{P}_{\mathrm{x}+5}=10 \mathrm{P}_{\mathrm{x}}-{ }_{5} \mathrm{P}_{\mathrm{x}} \\
& \text { Where: }{ }_{5} \mathrm{P}_{\mathrm{x}+5}=\text { population age } \mathrm{x}+5 \text { to } \mathrm{x}+9 \\
& { }_{10} \mathrm{P}_{\mathrm{x}}=\text { population age } \mathrm{x} \text { to } \mathrm{x}+9 \\
& { }_{5} \mathrm{P}_{\mathrm{x}}=\text { population at } \mathrm{x} \text { to } \mathrm{x}+4
\end{aligned}
$$

\section{United Nations Moving Average}

This method is appropriate for graduating errors inherent in age statistics i.e. it adjusts age data which is fraught with digit preference and age shift. It assumes that assumes that the expected age distribution is a smooth one.

$$
\begin{gathered}
\text { Si }=\frac{1}{16} \\
\left.\mathrm{P}_{i-2}+4 \mathrm{P}_{i-1}+10 \mathrm{P}_{i}+4 \mathrm{P}_{i+1}-\mathrm{P}_{i+2}\right) \\
\text { Si represents the smoothed population } \mathrm{x} \text { to } \mathrm{x}+4
\end{gathered}
$$

African Population Studies Vol 32, No. 3, Dec. 2018

Where $P_{i-2}, P_{i-1,}, P_{i}, P_{i+1}, P_{i+2}$ are the enumerated 5 -year age groups

\section{Strong smoothing formula}

The strong moving average was calculated using the formula below (Arriaga, 1968):

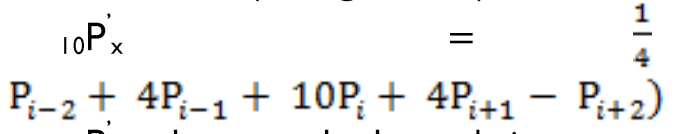

${ }_{10} P_{x}^{\prime}$ the smoothed population ages $x$ to $x+9$

\section{Percent error}

The percent errors in enumerated population were computed using the formula below:

$$
\mu=\mathrm{T}-\mathrm{S}
$$

Where $\mu=$ Error

$T=$ True population

$\mathrm{E}=$ Enumerated Population or

$\mathrm{S}=$ Smoothed Population

$$
\therefore \% \mu=\frac{T-S}{T} \times 100
$$


Table 1.1

Error Detection in Age-Sex Population Data of B urkina Faso in 1996 and 2006

\begin{tabular}{|c|c|c|c|c|c|c|c|c|c|c|c|c|c|c|}
\hline & & & Burki & Taso 199 & & & & & & & ina Faso 2 & 06 & & \\
\hline & Populati & & Age $R$ & & $\begin{array}{l}\text { Age R } \\
\text { Devia }\end{array}$ & $\begin{array}{l}\text { atio } \\
\text { ion }\end{array}$ & & & pulation & & Age Ratio & & $\begin{array}{r}\text { Age Ratio } \\
\text { De viation } \\
\end{array}$ & \\
\hline Age & Male & Female & Male & Female & Male & Female & $\begin{array}{l}\text { Sex } \\
\text { ratio }\end{array}$ & Male & Female & Male & Female & Male & Female & $\begin{array}{l}\text { Sex } \\
\text { ratio }\end{array}$ \\
\hline $0-4$ & 904230 & 890640 & & & & & 101.5 & 1239190 & 1221500 & & & & & 101.4 \\
\hline $5-9$ & 922480 & 901020 & 112.9 & 114.8 & 12.9 & 14.8 & 102.4 & 1180370 & 1150420 & 94.9 & 94.4 & -5.1 & -5.6 & 102.6 \\
\hline$\overline{10-14}$ & 729440 & 679640 & 96.5 & 92.0 & -3.5 & -8.0 & 107.3 & 908210 & 851690 & 103.2 & 102.5 & 3.2 & 2.5 & $\overline{106.6}$ \\
\hline$\overline{15-19}$ & 588980 & 576700 & 101.9 & 102.1 & 1.9 & 2.1 & 102.1 & 724700 & 777350 & 104.8 & 102.0 & 4.8 & 2.0 & 93.2 \\
\hline $20-24$ & 426080 & 449590 & 91.0 & 92.2 & -9.0 & -7.8 & 94.8 & 539460 & 666370 & 88.9 & 95.8 & -11.1 & -4.2 & 81.0 \\
\hline $25-29$ & 347210 & 398170 & 96.7 & 101.7 & -3.3 & 1.7 & 87.2 & 455300 & 573940 & 101.7 & 105.8 & 1.7 & 5.8 & $\overline{79.3}$ \\
\hline $30-34$ & 291820 & 333700 & 101.0 & 100.4 & 1.0 & 0.4 & 87.4 & 366900 & 438650 & 99.4 & 93.9 & -0.6 & -6.1 & $\overline{83.6}$ \\
\hline $35-39$ & 230760 & 266720 & 96.1 & 95.7 & -3.9 & -4.3 & 86.5 & 303380 & 362030 & 105.9 & 101.9 & 5.9 & 1.9 & $\overline{83.8}$ \\
\hline$\overline{40-44}$ & 188340 & 223420 & 97.6 & 102.5 & -2.4 & 2.5 & 84.3 & 253430 & 302740 & 91.6 & 86.7 & -8.4 & -13.3 & 83.7 \\
\hline $45-49$ & 155120 & 169050 & 96.6 & 89.6 & -3.4 & -10.4 & 91.8 & 196020 & 236090 & 96.8 & 101.7 & -3.2 & 1.7 & 83.0 \\
\hline $50-54$ & 132980 & 153960 & 102.0 & 112.1 & 2.0 & 12.1 & 86.4 & 169130 & 195080 & 97.2 & 103.7 & -2.8 & 3.7 & 86.7 \\
\hline $55-59$ & 105640 & 105590 & 91.4 & 81.9 & -8.6 & -18.1 & 100.0 & 132350 & 141800 & 88.6 & 81.0 & -11.4 & -19.0 & 93.3 \\
\hline $60-64$ & 98140 & 103760 & 114.2 & 120.7 & 14.2 & 20.7 & 94.6 & 111470 & 128880 & 102.1 & 111.5 & 2.1 & 11.5 & $\overline{86.5}$ \\
\hline $65-69$ & 66200 & 66340 & 86.6 & 80.6 & -13.4 & -19.4 & 99.8 & 80340 & 82990 & 93.1 & 94.0 & -6.9 & -6.0 & 96.8 \\
\hline $70-74$ & 54810 & 60780 & & & & & 90.2 & 63560 & 72360 & & & & & 87.8 \\
\hline
\end{tabular}

Mean Age Ratio $($ Male $)=5.68$

Mean Age Ratio $($ Female $)=8.74$

Age-sex accuracy inde $x=31.8$
Mean Age Ratio (Male) $=\mathbf{4 . 8 0}$

Mean Age Ratio $($ Female $)=\mathbf{5 . 9 5}$

Age-sex accuracy index $=\mathbf{2 8 . 6}$ 


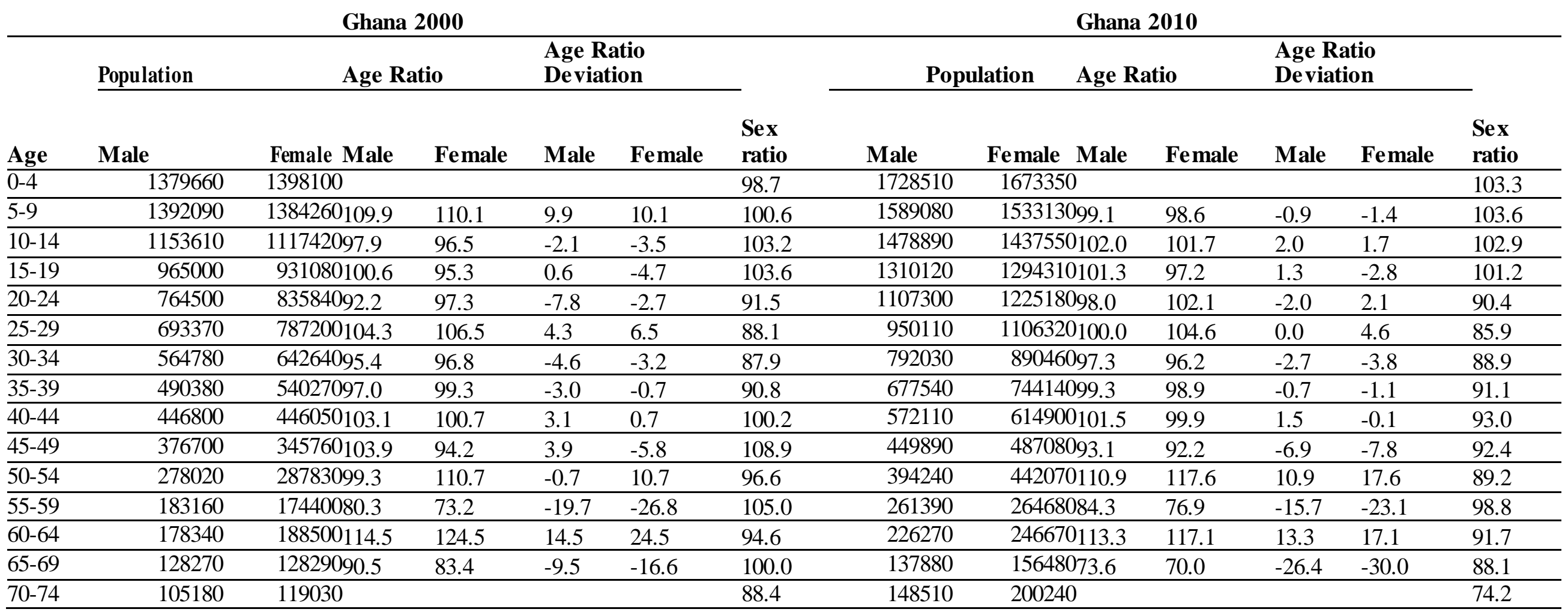

Mean Age Ratio $($ Male $)=5.98$

Mean Age Ratio $($ Female $)=8.32$

Age-sex accuracy inde $x=34.7$
Mean Age Ratio $($ Male $)=\quad 6.02$

Mean Age Ratio $($ Female $)=8.09$

Age-sex accuracy inde $x=28.8$ 


\begin{tabular}{|c|c|c|c|c|c|c|c|c|c|c|c|c|c|c|}
\hline & & & Came & oon 1987 & & & & & & & meroon 2 & 05 & & \\
\hline & Populat & & Age $R$ & tio & $\begin{array}{l}\text { Age R } \\
\text { Devia }\end{array}$ & & & & pulation & & ge Ratio & & $\begin{array}{l}\text { ge Ratio } \\
\text { Deviation }\end{array}$ & \\
\hline Age & Male & Female & Male & Female & Male & Female & $\begin{array}{l}\text { Sex } \\
\text { Ratio } \\
\end{array}$ & Male & Female & Male & Female & Male & Female & atio \\
\hline $0-4$ & 829510 & 807600 & & & & & 102.7 & 1514280 & 1474210 & & & & & 102.7 \\
\hline $5-9$ & 707020 & 677380 & 101.7 & 102.7 & 1.7 & 2.7 & 104.4 & 1272380 & 1244730 & 96.6 & 98.1 & -3.4 & -1.9 & 102.2 \\
\hline 10-14 & 561530 & 511000 & 99.0 & 89.7 & -1.0 & -10.3 & 109.9 & 1120080 & 1063700 & 99.5 & 94.4 & -0.5 & -5.6 & 105.3 \\
\hline 15-19 & 427750 & 462500 & 95.5 & 101.9 & -4.5 & 1.9 & 92.5 & 979880 & 1008600 & 102.9 & 102.5 & 2.9 & 2.5 & 97.2 \\
\hline $20-24$ & 333850 & 396840 & 91.6 & 96.7 & -8.4 & -3.3 & 84.1 & 785340 & 904650 & 96.0 & 103.1 & -4.0 & 3.1 & 86.8 \\
\hline $25-29$ & 301540 & 358230 & 102.7 & 104.8 & 2.7 & 4.8 & 84.2 & 657080 & 746220 & 99.2 & 101.2 & -0.8 & 1.2 & 88.1 \\
\hline $30-34$ & 253170 & 286630 & 101.6 & 101.1 & 1.6 & 1.1 & 88.3 & 539230 & 569900 & 100.3 & 96.2 & 0.3 & -3.8 & 94.6 \\
\hline $35-39$ & 196980 & 208890 & 91.9 & 86.2 & -8.1 & -13.8 & 94.3 & 418600 & 438990 & 93.4 & 93.7 & -6.6 & -6.3 & 95.4 \\
\hline $40-44$ & 175740 & 197800 & 101.0 & 107.7 & 1.0 & 7.7 & 88.8 & 357150 & 367390 & 100.3 & 101.0 & 0.3 & 1.0 & 97.2 \\
\hline $45-49$ & 151040 & 158400 & 95.6 & 91.1 & -4.4 & -8.9 & 95.4 & 293620 & 288670 & 98.9 & 94.9 & -1.1 & -5.1 & 101.7 \\
\hline 50-54 & 140390 & 149950 & 112.8 & 118.6 & 12.8 & 18.6 & 93.6 & 236700 & 241000 & 103.7 & 110.8 & 3.7 & 10.8 & 98.2 \\
\hline $55-59$ & 97910 & 94480 & 86.8 & 78.0 & -13.2 & -22.0 & 103.6 & 162720 & 146390 & 86.1 & 74.7 & -13.9 & -25.3 & 111.2 \\
\hline 60-64 & 85330 & 92300 & 112.8 & 125.0 & 12.8 & 25.0 & 92.4 & 141070 & 150740 & 106.8 & 117.6 & 6.8 & 17.6 & 93.6 \\
\hline $65-69$ & 53350 & 53170 & 82.7 & 75.9 & -17.3 & -24.1 & 100.3 & 101540 & 109920 & 90.9 & 89.7 & -9.1 & -10.3 & 92.4 \\
\hline $70-74$ & 43690 & 47870 & & & & & 91.3 & 82450 & 94440 & & & & & 87.3 \\
\hline
\end{tabular}

Mean Age Ratio $($ Male $)=6.39$

Mean Age Ratio $($ Female $)=\mathbf{1 0 . 3 0}$

Age-sex accuracy index $=\mathbf{3 8 . 3}$
Mean Age Ratio (Male) $=3.81$

Mean Age Ratio (Female) $=6.75$

Age-sex accuracy inde $x=27.9$ 
Error Detection in Age-Sex Population Data of Mali in 1998 and 2009

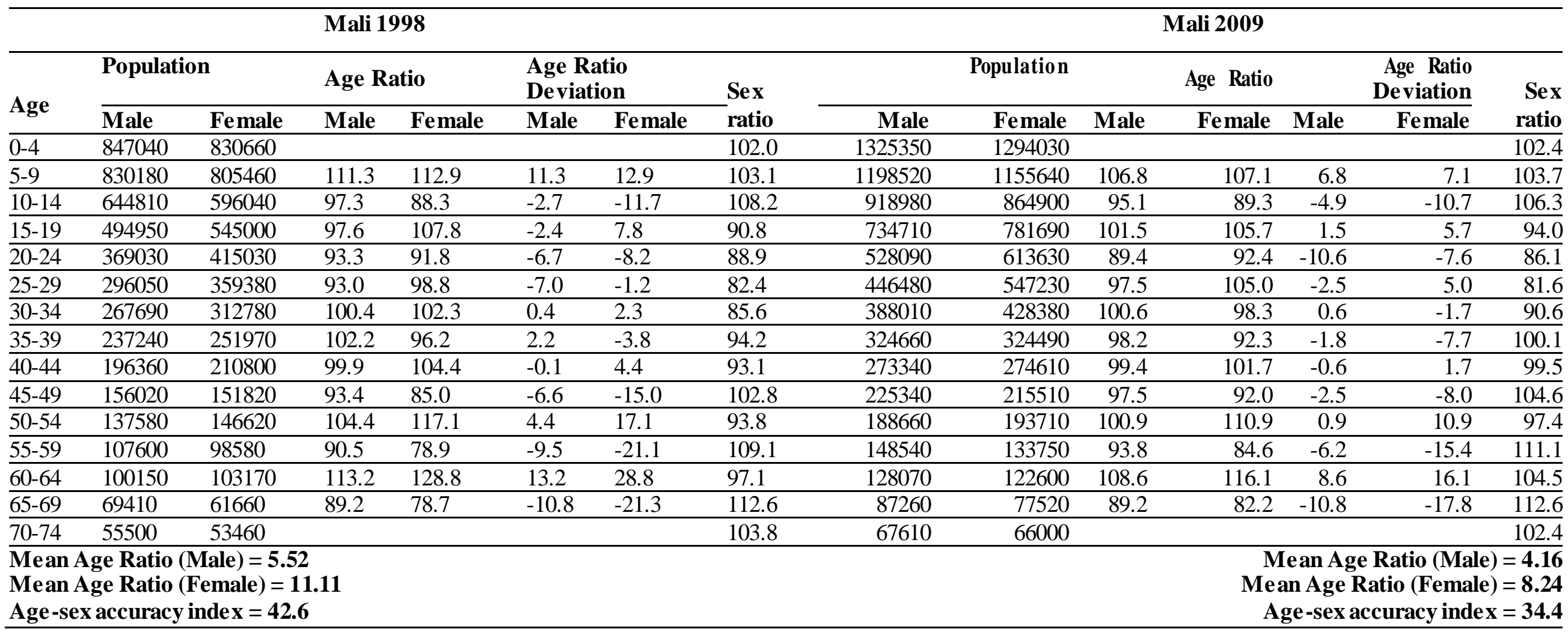




\begin{tabular}{|c|c|c|c|c|c|c|c|c|c|c|c|c|c|c|}
\hline \multirow[b]{3}{*}{ Age } & \multicolumn{7}{|c|}{ Senegal 1988} & \multicolumn{7}{|c|}{ Sene gal 2002} \\
\hline & \multicolumn{2}{|c|}{ Population } & \multicolumn{2}{|c|}{ Age Ratio } & \multicolumn{2}{|c|}{$\begin{array}{l}\text { Age Ratio } \\
\text { Deviation }\end{array}$} & \multirow{2}{*}{$\begin{array}{l}\text { Sex } \\
\text { ratio }\end{array}$} & \multicolumn{3}{|c|}{ Population } & Age Ratio & \multicolumn{2}{|r|}{$\begin{array}{r}\text { Age Ratio } \\
\text { Deviation }\end{array}$} & \multirow{2}{*}{$\begin{array}{l}\text { Sex } \\
\text { ratio }\end{array}$} \\
\hline & Male & Female & Male & Female & Male & Female & & Male & Female & Male & Female & Male & Female & \\
\hline $0-4$ & 666100 & 661530 & & & & & 100.7 & 732590 & 724590 & & & & & 101.1 \\
\hline 5 & 551090 & 578140 & 102.8 & 107.4 & 2.8 & 7.4 & 95.3 & 741530 & 733170 & 106.7 & 107.1 & 6.7 & 7.1 & $\overline{101.1}$ \\
\hline $10-14$ & 406520 & 415120 & 91.8 & 86.0 & -8.2 & -14.0 & 97.9 & 657970 & 644260 & 100.7 & 95.9 & 0.7 & -4.1 & 102.1 \\
\hline $15-19$ & 334430 & 387740 & 98.7 & 107.2 & -1.3 & 7.2 & 86.3 & 565640 & 611100 & 101.1 & 108.6 & 1.1 & 8.6 & 92.6 \\
\hline $20-24$ & 271180 & 308430 & 92.6 & 90.1 & -7.4 & -9.9 & 87.9 & 461220 & 480890 & 99.4 & 95.6 & -0.6 & -4.4 & 95.9 \\
\hline $25-29$ & 251140 & 296740 & 109.3 & 116.7 & 9.3 & 16.7 & 84.6 & 362110 & 394810 & 96.1 & 97.8 & -3.9 & -2.2 & 91.7 \\
\hline $30-34$ & 188380 & 200010 & 90.6 & 83.1 & -9.4 & -16.9 & 94.2 & 292520 & 326440 & 99.7 & 100.2 & -0.3 & 0.2 & 89.6 \\
\hline $35-39$ & 164670 & 184760 & 111.4 & 117.0 & 11.4 & 17.0 & 89.1 & 224670 & 256590 & 90.2 & 93.5 & -9.8 & -6.5 & 87.6 \\
\hline $40-44$ & 107280 & 115950 & 77.7 & 77.6 & -22.3 & -22.4 & 92.5 & 205780 & 222700 & 107.9 & 107.6 & 7.9 & 7.6 & $\overline{92.4}$ \\
\hline $45-49$ & 111300 & 114090 & 114.7 & 115.0 & 14.7 & 15.0 & 97.6 & 156710 & 157410 & 91.0 & 86.0 & -9.0 & -14.0 & 99.6 \\
\hline $50-54$ & 86710 & 82450 & 89.2 & 84.7 & -10.8 & -15.3 & 105.2 & 138450 & 143430 & 113.6 & 118.2 & 13.6 & 18.2 & 96.5 \\
\hline 55-59 & 83010 & 80690 & 113.9 & 120.3 & 13.9 & 20.3 & 102.9 & 86960 & 85230 & 76.6 & 71.6 & -23.4 & -28.4 & 102.0 \\
\hline $60-64$ & 59060 & 51670 & 86.9 & 80.5 & -13.1 & -19.5 & 114.3 & 88630 & 94510 & 120.5 & 135.3 & 20.5 & 35.3 & 93.8 \\
\hline $65-69$ & 52960 & 47740 & 119.4 & 125.5 & 19.4 & 25.5 & 110.9 & 60120 & 54510 & 84.0 & 71.4 & -16.0 & -28.6 & 110.3 \\
\hline 70-74 & 29660 & 24420 & & & & & 121.5 & 54520 & 58210 & & & & & 93.7 \\
\hline
\end{tabular}

Mean Age Ratio $($ Male $)=10.29$

Mean Age Ratio $($ Female $)=14.79$

Age-sex accuracy inde $x=44.8$
Mean Age Ratio $($ Male $)=8.11$ Mean Age Ratio $($ Female $)=\mathbf{1 1 . 8 0}$ Age-sex accuracy inde $x=39.5$ 
African Population Studies Vol 32, No. 3, Dec. 2018

Table 1. MALE POPULATION OF BURKINA FASO, 1996

\begin{tabular}{|c|c|c|c|c|c|c|c|}
\hline AGE & REPORTED & $\begin{array}{c}\text { CARR IER } \\
\text { FARAG }\end{array}$ & K.KING & ARRIAGA & $\begin{array}{l}\text { UN MOVING } \\
\text { AVERA GE }\end{array}$ & $\begin{array}{c}\text { STRONG } \\
\text { MOVING } \\
\text { AVERAGE }\end{array}$ & \%ERROR \\
\hline $0-4$ & 904230 & & & 975356 & & 987697 & 8.45 \\
\hline $5-9$ & 922480 & & & 851354 & & 839013 & 9.95 \\
\hline 10-14 & 729440 & 729772 & 725049 & 725816 & 750621 & 694915 & 4.97 \\
\hline 15-19 & 588980 & 588648 & 593371 & 592604 & 577637 & 573745 & 2.66 \\
\hline $20-24$ & 426080 & 431172 & 436385 & 430251 & 436519 & 453742 & 6.10 \\
\hline $25-29$ & 347210 & 342118 & 336905 & 343039 & 345248 & 366922 & 5.37 \\
\hline 30-34 & 291820 & 287707 & 288154 & 286663 & 288479 & 288650 & 1.10 \\
\hline 35-39 & 230760 & 234873 & 234426 & 235917 & 232869 & 235087 & 1.84 \\
\hline $40-44$ & 188340 & 188504 & 189478 & 187930 & 187633 & 191962 & 1.89 \\
\hline $45-49$ & 155120 & 154956 & 153983 & 155530 & 156255 & 158855 & 2.35 \\
\hline $50-54$ & 132980 & 130272 & 130505 & 129868 & 130398 & 130694 & 1.75 \\
\hline $55-59$ & 105640 & 108348 & 108115 & 108752 & 109973 & 107939 & 2.13 \\
\hline 60-64 & 98140 & 92629 & 91726 & 91817 & 92561 & 88491 & 10.90 \\
\hline 65-69 & 66200 & 71711 & 72614 & 72523 & 71078 & 69708 & 5.03 \\
\hline $70-74$ & 54810 & & & 52868 & & 51588 & 6.25 \\
\hline $75-79$ & 30910 & & & 32852 & & 34132 & 9.44 \\
\hline \multirow[t]{2}{*}{$80+$} & 30850 & & & & & & \\
\hline & \multicolumn{6}{|c|}{ FEMALE POPULATION OF B URKINA FASO, 1996} & \\
\hline AGE & REPORTED & $\begin{array}{c}\text { CARR IER } \\
\text { FARAG }\end{array}$ & K.KING & ARRIAGA & $\begin{array}{c}\text { UN MOVING } \\
\text { AVERA GE }\end{array}$ & $\begin{array}{c}\text { STRONG } \\
\text { MOVING } \\
\text { AVERAGE }\end{array}$ & \%ERROR \\
\hline $0-4$ & 890640 & & & 968026 & & 970363 & 8.22 \\
\hline $5-9$ & 901020 & & & 823634 & & 821297 & 9.71 \\
\hline 10-14 & 679640 & 686757 & 687164 & 684523 & 710441 & 678757 & 0.13 \\
\hline $15-19$ & 576700 & 569583 & 569176 & 571817 & 561546 & 568851 & 1.38 \\
\hline $20-24$ & 449590 & 462890 & 464875 & 461516 & 461378 & 468667 & 4.07 \\
\hline $25-29$ & 398170 & 384870 & 382885 & 386244 & 391965 & 391536 & 1.69 \\
\hline
\end{tabular}


African Population Studies Vol 32, No. 3, Dec. 2018

\begin{tabular}{|c|c|c|c|c|c|c|c|}
\hline 30-34 & 333700 & 329021 & 328666 & 327845 & 332722 & 322837 & 3.36 \\
\hline 35-39 & 266720 & 271399 & 271754 & 272575 & 270529 & 268281 & 0.58 \\
\hline $40-44$ & 223420 & 216732 & 217539 & 215976 & 218101 & 218561 & 2.22 \\
\hline $45-49$ & 169050 & 175738 & 174931 & 176494 & 176732 & 179763 & 5.96 \\
\hline $50-54$ & 153960 & 143289 & 143673 & 142768 & 144436 & 144522 & 6.53 \\
\hline 55-59 & 105590 & 116261 & 115877 & 116783 & 115712 & 117410 & 10.07 \\
\hline $60-64$ & 103760 & 96041 & 95536 & 95305 & 94411 & 93971 & 10.42 \\
\hline $65-69$ & 66340 & 74059 & 74564 & 74795 & 74061 & 73484 & 9.72 \\
\hline $70-74$ & 60780 & & & 55213 & & 54563 & 11.39 \\
\hline 75-79 & 30990 & & & 36557 & & 37207 & 16.71 \\
\hline $80+$ & 44180 & & & & & & \\
\hline \multicolumn{8}{|c|}{ Table 2. MALE POPULATION OF B URKINA FASO, 2006} \\
\hline AGE & REPORTED & $\begin{array}{c}\text { CARRIER } \\
\text { FARAG }\end{array}$ & K.KING & ARRIAGA & $\begin{array}{c}\text { UN MOVING } \\
\text { AVERAGE }\end{array}$ & $\begin{array}{c}\text { STRONG } \\
\text { MOVING } \\
\text { AVERA GE }\end{array}$ & \%ERROR \\
\hline $0-4$ & 1239190 & & & 1314299 & & 1321775 & 6.25 \\
\hline $5-9$ & 1180370 & & & 1105261 & & 1097785 & 7.52 \\
\hline 10-14 & 908210 & 906796 & 905505 & 902411 & 932733 & 883877 & 2.75 \\
\hline 15-19 & 724700 & 726114 & 727405 & 730499 & 712626 & 720377 & 0.60 \\
\hline 20-24 & 539460 & 552512 & 557544 & 551010 & 552468 & 569520 & 5.28 \\
\hline $25-29$ & 455300 & 442248 & 437216 & 443750 & 446898 & 461386 & 1.32 \\
\hline $30-34$ & 366900 & 368314 & 369222 & 367063 & 369427 & 367768 & 0.24 \\
\hline 35-39 & 303380 & 301966 & 301058 & 303218 & 303988 & 301002 & 0.79 \\
\hline 40-44 & 253430 & 247095 & 247775 & 246257 & 249742 & 246306 & 2.89 \\
\hline $45-49$ & 196020 & 202355 & 201675 & 203193 & 200919 & 202938 & 3.41 \\
\hline 50-54 & 169130 & 166724 & 166843 & 166045 & 164993 & 164844 & 2.60 \\
\hline 55-59 & 132350 & 134756 & 134638 & 135435 & 135596 & 133959 & 1.20 \\
\hline $60-64$ & 111470 & 108969 & 108454 & 108067 & 108298 & 106277 & 4.89 \\
\hline 65-69 & 80340 & 82841 & 83356 & 83743 & 83377 & 82435 & 2.54 \\
\hline $70-74$ & & 63560 & & 60965 & & 60432 & 5.18 \\
\hline
\end{tabular}


FEMALE POPULATION OF B URKINA FASO, 2006

\begin{tabular}{|c|c|c|c|c|c|c|c|}
\hline AGE & REPORTED & $\begin{array}{c}\text { CARRIER } \\
\text { FARAG }\end{array}$ & K.KING & ARRIAGA & $\begin{array}{l}\text { UN MOVING } \\
\text { AVERA GE }\end{array}$ & $\begin{array}{l}\text { STRONG } \\
\text { MOVING } \\
\text { AVERAGE }\end{array}$ & \%ERROR \\
\hline $0-4$ & 1221500 & & & 1293576 & & 1284639 & 4.91 \\
\hline $5-9$ & 1150420 & & & 1078344 & & 1087281 & 5.81 \\
\hline 10-14 & 851690 & 880386 & 885246 & 877868 & 896257 & 899782 & 5.34 \\
\hline 15-19 & 777350 & 748654 & 743794 & 751173 & 757586 & 761577 & 2.07 \\
\hline $20-24$ & 666370 & 675072 & 671928 & 672988 & 673658 & 644278 & 3.43 \\
\hline $25-29$ & 573940 & 565238 & 568383 & 567322 & 563756 & 543126 & 5.67 \\
\hline $30-34$ & 438650 & 441911 & 444183 & 440479 & 447579 & 447628 & 2.01 \\
\hline 35-39 & 362030 & 358769 & 356498 & 360201 & 360989 & 369835 & 2.11 \\
\hline $40-44$ & 302740 & 298457 & 298403 & 297155 & 299134 & 295515 & 2.44 \\
\hline $45-49$ & 236090 & 240373 & 240428 & 241675 & 240522 & 240163 & 1.70 \\
\hline 50-54 & 195080 & 188005 & 188875 & 187272 & 189421 & 191298 & 1.98 \\
\hline 55-59 & 141800 & 148875 & 148005 & 149608 & 149673 & 153161 & 7.42 \\
\hline $60-64$ & 128880 & 120315 & 119930 & 119386 & 120033 & 119365 & 7.97 \\
\hline 65-69 & 82990 & 91555 & 91940 & 92484 & 90779 & 91882 & 9.68 \\
\hline 70-74 & 72360 & & & 67756 & & 67311 & 7.50 \\
\hline $75-79$ & 40600 & & & 45204 & & 45649 & 11.06 \\
\hline
\end{tabular}

$80+\quad 56940$

Table 3. MALE POPULATION OF GHANA, 2000

\begin{tabular}{|c|c|c|c|c|c|c|c|}
\hline AGE & REPORTED & $\begin{array}{l}\text { CARRIER } \\
\text { FARAG }\end{array}$ & K.KING & ARRIAGA & $\begin{array}{l}\text { UN MOVING } \\
\text { AVERAGE }\end{array}$ & $\begin{array}{l}\text { STRONG } \\
\text { MOVING } \\
\text { AVERA GE }\end{array}$ & \%ERROR \\
\hline $0-4$ & 1379660 & & & 1467201 & & 1478091 & 6.66 \\
\hline $5-9$ & 1392090 & & & 1304549 & & 1293659 & 7.61 \\
\hline$\overline{10-14}$ & 1153610 & 1144198 & 1141423 & & & 3.55 & \\
\hline
\end{tabular}


African Population Studies Vol 32, No. 3, Dec. 2018

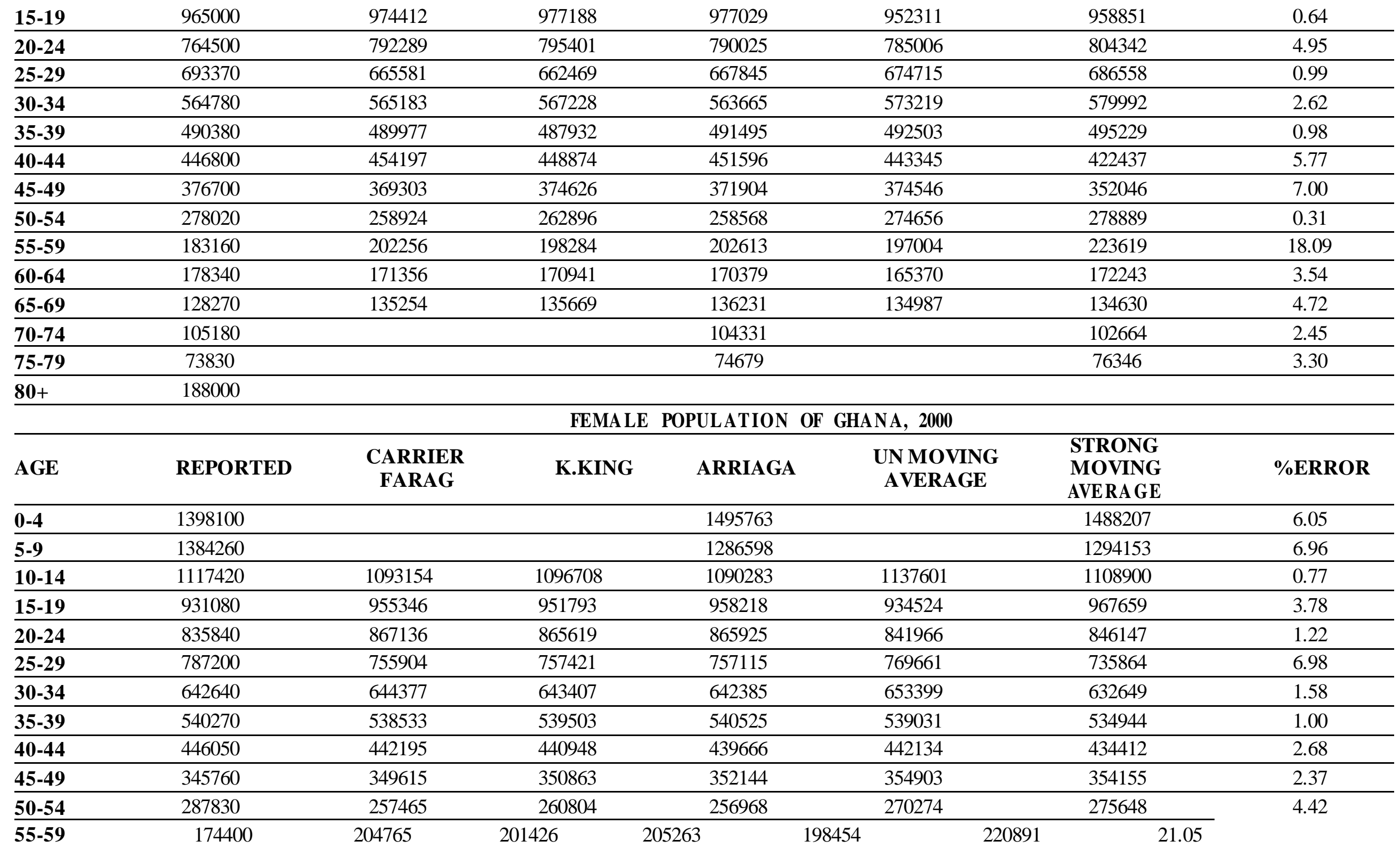


African Population Studies Vol 32, No. 3, Dec. 2018

\begin{tabular}{|c|c|c|c|c|c|c|c|}
\hline $60-64$ & 188500 & 175936 & 175416 & 175029 & 168056 & 174954 & 7.74 \\
\hline 65-69 & 128290 & 140854 & 141374 & 141761 & 141734 & 139058 & 7.74 \\
\hline 70-74 & 119030 & & & 110038 & & 108030 & 10.18 \\
\hline 75-79 & 70870 & & & 79862 & & 81870 & 13.44 \\
\hline
\end{tabular}

80+ 182100

Table 4. MALE POPULATION OF GHANA, 2010

\begin{tabular}{|c|c|c|c|c|c|c|c|}
\hline AGE & REPORTED & $\begin{array}{c}\text { CARRIER } \\
\text { FARAG }\end{array}$ & K.KING & ARRIAGA & $\begin{array}{l}\text { UN MOVING } \\
\text { AVERAGE }\end{array}$ & $\begin{array}{l}\text { STRONG } \\
\text { MOVING } \\
\text { AVERAGE }\end{array}$ & \% ERROR \\
\hline $0-4$ & 1728510 & & & 1716408 & & 1734223 & 0.33 \\
\hline $5-9$ & 1589080 & & & 1601182 & & 1583367 & 0.36 \\
\hline 10-14 & 1478890 & 1477691 & 1473266 & 1477496 & 1471868 & 1431454 & 3.31 \\
\hline 15-19 & 1310120 & 1311319 & 1315744 & 1311514 & 1306673 & 1274257 & 2.81 \\
\hline $20-24$ & 1107300 & 1110918 & 1111170 & 1108175 & 1115188 & 1109264 & 0.18 \\
\hline $25-29$ & 950110 & 946492 & 946240 & 949235 & 944423 & 959207 & 0.95 \\
\hline 30-34 & 792030 & 798887 & 799498 & 796576 & 796968 & 805686 & 1.69 \\
\hline 35-39 & 677540 & 670683 & 670072 & 672994 & 676998 & 681069 & 0.52 \\
\hline 40-44 & 572110 & 562381 & 561871 & 560180 & 565284 & 564289 & 1.39 \\
\hline $45-49$ & 449890 & 459619 & 460129 & 461820 & 464086 & 465623 & 3.38 \\
\hline 50-54 & 394240 & 369868 & 368931 & 367370 & 374321 & 370464 & 6.42 \\
\hline 55-59 & 261390 & 285762 & 286699 & 288260 & 281761 & 295874 & 11.65 \\
\hline $60-64$ & 226270 & 205046 & 208198 & 204760 & 207314 & 224940 & 0.59 \\
\hline 65-69 & 137880 & 159104 & 155953 & 159390 & 157961 & 175642 & 21.50 \\
\hline $70-74$ & 148510 & & & 127770 & & 134914 & 10.08 \\
\hline $75-79$ & 89160 & & & 109900 & & 102756 & 13.23 \\
\hline \multirow[t]{2}{*}{ 80+ } & 123880 & & & & & & \\
\hline & \multicolumn{7}{|c|}{ FEMALE POPULATION OF GHANA, 2010} \\
\hline AGE & REPORTED & $\begin{array}{l}\text { CARRIER } \\
\text { FARAG }\end{array}$ & K.KING & ARRIAGA & $\begin{array}{l}\text { UN MOVING } \\
\text { AVERAGE }\end{array}$ & $\begin{array}{l}\text { STRONG } \\
\text { MOVING } \\
\text { AVERAGE }\end{array}$ & \%ERROR \\
\hline
\end{tabular}


African Population Studies Vol 32, No. 3, Dec. 2018

\begin{tabular}{|c|c|c|c|c|c|c|c|}
\hline $0-4$ & 1673350 & & & 1665662 & & 1663851 & 0.57 \\
\hline $5-9$ & 1533130 & & & 1540818 & & 1542629 & 0.62 \\
\hline $10-14$ & 1437550 & 1420310 & 1420616 & 1419069 & 1424171 & 1421317 & 1.14 \\
\hline 15-19 & 1294310 & 1311550 & 1311244 & 1312791 & 1309661 & 1299555 & 0.40 \\
\hline $20-24$ & 1225180 & 1240486 & 1234329 & 1240507 & 1220394 & 1184832 & 3.41 \\
\hline $25-29$ & 1106320 & 1091014 & 1097171 & 1090993 & 1092957 & 1048278 & 5.54 \\
\hline $30-34$ & 890460 & 893638 & 894145 & 890723 & 904148 & 897272 & 0.76 \\
\hline 35-39 & 744140 & 740962 & 740455 & 743878 & 741840 & 760393 & 2.14 \\
\hline $40-44$ & 614900 & 608528 & 608981 & 606118 & 608834 & 617815 & 0.47 \\
\hline $45-49$ & 487080 & 493452 & 492999 & 495862 & 505616 & 506303 & 3.80 \\
\hline 50-54 & 442070 & 397560 & 397052 & 395143 & 410386 & 400134 & 10.48 \\
\hline $55-59$ & 264680 & 309190 & 309698 & 311607 & 297388 & 321683 & 17.72 \\
\hline $60-64$ & 246670 & 221818 & 226020 & 221518 & 219314 & 248746 & 0.83 \\
\hline 65-69 & 156480 & 181332 & 177130 & 181632 & 185773 & 203512 & 23.11 \\
\hline 70-74 & 200240 & & & 159752 & & 169355 & 18.24 \\
\hline $75-79$ & 115390 & & & 155878 & & 146275 & 21.11 \\
\hline
\end{tabular}

$\mathbf{8 0 +} 194030$

Table 5. MALE POPULATION OF CAMEROON, 1987

\begin{tabular}{|c|c|c|c|c|c|c|c|}
\hline AGE & REPORTED & $\begin{array}{c}\text { CARRIER } \\
\text { FARAG }\end{array}$ & K.KING & ARRIAGA & $\begin{array}{c}\text { UN MOVING } \\
\text { A VERAGE }\end{array}$ & $\begin{array}{l}\text { STRONG } \\
\text { MOVING } \\
\text { AVERAGE }\end{array}$ & \%ERROR \\
\hline $0-4$ & 829510 & & & 844728 & & 844044 & 1.72 \\
\hline $5-9$ & 707020 & & & 691802 & & 692486 & 2.10 \\
\hline 10-14 & 561530 & 549018 & 550961 & 546933 & 561939 & 549077 & 2.27 \\
\hline 15-19 & 427750 & 440262 & 438319 & 442347 & 428154 & 446416 & 4.18 \\
\hline $20-24$ & 333850 & 348863 & 351391 & 347877 & 340060 & 356360 & 6.32 \\
\hline $25-29$ & 301540 & 286527 & 283999 & 287513 & 296172 & 293684 & 2.67 \\
\hline 30-34 & 253170 & 243740 & 244363 & 243074 & 251012 & 242232 & 4.52 \\
\hline 35-39 & 196980 & 206410 & 205787 & & & & 3.67 \\
\hline
\end{tabular}


African Population Studies Vol 32, No. 3, Dec. 2018

\begin{tabular}{|c|c|c|c|c|c|c|c|}
\hline 40-44 & 175740 & 176353 & 176631 & 175904 & 172245 & 174136 & 0.92 \\
\hline $45-49$ & 151040 & 150427 & 150149 & 150876 & 155002 & 147745 & 2.23 \\
\hline $50-54$ & 140390 & 131867 & 130906 & 131138 & 133664 & 124262 & 12.98 \\
\hline 55-59 & 97910 & 106433 & 107394 & 107162 & 104849 & 101691 & 3.72 \\
\hline 60-64 & 85330 & 80934 & 80374 & 79901 & 79641 & 79286 & 7.62 \\
\hline 65-69 & 53350 & 57746 & 58306 & 58779 & 58350 & 59208 & 9.89 \\
\hline $70-74$ & 43690 & & & 39549 & & 40025 & 9.16 \\
\hline $75-79$ & 18070 & & & 22211 & & 21735 & 16.86 \\
\hline
\end{tabular}

FEMALE POPULATION OF CAMEROON, 1987

\begin{tabular}{|c|c|c|c|c|c|c|c|}
\hline AGE & REPORTED & $\begin{array}{c}\text { CARRIER } \\
\text { FARAG }\end{array}$ & K.KING & ARRIAGA & $\begin{array}{c}\text { UN MOVING } \\
\text { AVERAGE }\end{array}$ & $\begin{array}{l}\text { STRONG } \\
\text { MOVING } \\
\text { AVERAGE }\end{array}$ & \%ERROR \\
\hline $0-4$ & 807600 & & & 818635 & & 809500 & 0.23 \\
\hline $5-9$ & 677380 & & & 666345 & & 675480 & 0.28 \\
\hline 10-14 & 511000 & 527804 & 532369 & 526264 & 529068 & 549107 & 6.94 \\
\hline 15-19 & 462500 & 445696 & 441131 & 447236 & 451297 & 460966 & 0.33 \\
\hline 20-24 & 396840 & 409328 & 407409 & 408265 & 403356 & 389111 & 1.99 \\
\hline $25-29$ & 358230 & 345742 & 347661 & 346805 & 352799 & 329574 & 8.69 \\
\hline 30-34 & 286630 & 270960 & 272689 & 270185 & 283759 & 275458 & 4.06 \\
\hline 35-39 & 208890 & 224560 & 222831 & 225335 & 219374 & 231698 & 9.84 \\
\hline 40-44 & 197800 & 193792 & 193793 & 193219 & 188161 & 190998 & 3.56 \\
\hline $45-49$ & 158400 & 162408 & 162407 & 162981 & 166977 & 159363 & 0.60 \\
\hline 50-54 & 149950 & 135839 & 135386 & 135119 & 138808 & 131951 & 13.64 \\
\hline 55-59 & 94480 & 108591 & 109044 & 109311 & 106389 & 107002 & 11.70 \\
\hline $60-64$ & 92300 & 84596 & 83916 & 83519 & 82236 & 83016 & 11.18 \\
\hline $65-69$ & 53170 & 60874 & 61554 & 61951 & 61264 & 61946 & 14.17 \\
\hline 70-74 & 47870 & & & 41968 & & 42091 & 13.73 \\
\hline $75-79$ & 17670 & & & & & & \\
\hline
\end{tabular}


Table 6. MALE POPULATION OF CAMEROON, 2005

\begin{tabular}{|c|c|c|c|c|c|c|c|}
\hline AGE & REPORTED & $\begin{array}{l}\text { CARR IER } \\
\text { FARAG }\end{array}$ & K.KING & ARRIAGA & $\begin{array}{c}\text { UN MOVING } \\
\text { AVERAGE }\end{array}$ & $\begin{array}{l}\text { STRONG } \\
\text { MOVING } \\
\text { AVERAGE }\end{array}$ & \%ERROR \\
\hline $0-4$ & 1514280 & & & 1480383 & & 1489718 & 1.65 \\
\hline $5-9$ & 1272380 & & & 1306278 & & 1296942 & 1.89 \\
\hline 10-14 & 1120080 & 1136215 & 1133995 & 1133388 & 1119389 & 1109304 & 0.97 \\
\hline 15-19 & 979880 & 963745 & 965965 & 966573 & 968189 & 947358 & 3.43 \\
\hline 20-24 & 785340 & 791753 & 792593 & 788990 & 796371 & 789594 & 0.54 \\
\hline $25-29$ & 657080 & 650667 & 649827 & 653430 & 654413 & 660398 & 0.50 \\
\hline $30-34$ & 539230 & 526406 & 528393 & 524695 & 534533 & 536188 & 0.57 \\
\hline 35-39 & 418600 & 431424 & 429437 & 433135 & 426301 & 441965 & 5.29 \\
\hline $40-44$ & 357150 & 360819 & 360286 & 359125 & 352778 & 357749 & 0.17 \\
\hline $45-49$ & 293620 & 289951 & 290484 & 291645 & 295643 & 290991 & 0.90 \\
\hline $50-54$ & 236700 & 224218 & 225220 & 223250 & 230884 & 230137 & 2.85 \\
\hline $55-59$ & 162720 & 175202 & 174200 & 176170 & 171445 & 182762 & 10.97 \\
\hline $60-64$ & 141070 & 138693 & 138401 & 137566 & 134287 & 140270 & 0.57 \\
\hline 65-69 & 101540 & 103917 & 104209 & 105044 & 106458 & 106295 & 4.47 \\
\hline $70-74$ & 82450 & & & 75861 & & 76124 & 8.31 \\
\hline 75-79 & 43430 & & & 50019 & & 49756 & 12.71 \\
\hline \multirow[t]{2}{*}{$80+$} & 52630 & & & & & & \\
\hline & \multicolumn{7}{|c|}{ FEMALE POPULATION OF CAMEROON, 2005} \\
\hline AGE & REPORTED & $\begin{array}{l}\text { CARR IER } \\
\text { FARAG }\end{array}$ & K.KING & ARRIAGA & $\begin{array}{c}\text { UN MOVING } \\
\text { AVERAGE }\end{array}$ & $\begin{array}{l}\text { STRONG } \\
\text { MOVING } \\
\text { AVERAGE }\end{array}$ & \%ERROR \\
\hline $0-4$ & 1474210 & & & 1449684 & & 1443733 & 2.11 \\
\hline $5-9$ & 1244730 & & & 1269256 & & 1275207 & 2.39 \\
\hline 10-14 & 1063700 & 1100688 & 1102904 & 1098213 & 1079466 & 1111451 & 4.30 \\
\hline $15-19$ & 1008600 & 971612 & 969396 & & & & \\
\hline
\end{tabular}


African Population Studies Vol 32, No. 3, Dec. 2018

\begin{tabular}{|c|c|c|c|c|c|c|c|}
\hline $20-24$ & 904650 & 899505 & 891898 & 896493 & 902011 & 844460 & 7.13 \\
\hline $25-29$ & 746220 & 751365 & 758972 & 754377 & 744551 & 717084 & 4.06 \\
\hline $30-34$ & 569900 & 562376 & 566621 & 560597 & 572988 & 582939 & 2.24 \\
\hline 35-39 & 438990 & 446514 & 442269 & 448293 & 444011 & 475075 & 7.60 \\
\hline $40-44$ & 367390 & 367092 & 366874 & 365120 & 360853 & 368504 & 0.30 \\
\hline $45-49$ & 288670 & 288968 & 289186 & 290940 & 295930 & 294089 & 1.84 \\
\hline $50-54$ & 241000 & 215945 & 218408 & 215450 & 227007 & 230324 & 4.64 \\
\hline $55-59$ & 146390 & 171445 & 168983 & 171940 & 164517 & 183491 & 20.22 \\
\hline 60-64 & 150740 & 146523 & 145627 & 145445 & 137325 & 144675 & 4.19 \\
\hline 65-69 & 109920 & 114137 & 115033 & 115215 & 117833 & 112531 & 2.32 \\
\hline 70-74 & 94440 & & & 85710 & & 83889 & 12.58 \\
\hline 75-79 & 48200 & & & 56930 & & 58751 & 17.96 \\
\hline
\end{tabular}

Table 7. MALE POPULATION OF MALI, 1988

\begin{tabular}{|c|c|c|c|c|c|c|c|}
\hline AGE & REPORTED & $\begin{array}{c}\text { CARRIER } \\
\text { FARAG }\end{array}$ & K.KING & ARRIAGA & $\begin{array}{c}\text { UN MOVING } \\
\text { AVERAGE }\end{array}$ & $\begin{array}{l}\text { STRONG } \\
\text { MOVING } \\
\text { AVERAGE }\end{array}$ & \% ERROR \\
\hline $0-4$ & 847040 & & & 908408 & & 915872 & 7.52 \\
\hline $5-9$ & 830180 & & & 768812 & & 761348 & 9.04 \\
\hline 10-14 & 644810 & 635479 & 633139 & 631831 & 658284 & 613730 & 5.06 \\
\hline 15-19 & 494950 & 504281 & 506621 & 507929 & 492414 & 500643 & 1.14 \\
\hline $20-24$ & 369030 & 366266 & 372217 & 365664 & 371363 & 394219 & 6.39 \\
\hline $25-29$ & 296050 & 298814 & 292863 & 299416 & 298449 & 323051 & 8.36 \\
\hline 30-34 & 267690 & 272469 & 272009 & 271850 & 265292 & 265426 & 0.85 \\
\hline 35-39 & 237240 & 232461 & 232921 & 233080 & 236033 & 223384 & 6.20 \\
\hline $40-44$ & 196360 & 192057 & 192424 & 191480 & 195711 & 190037 & 3.33 \\
\hline $45-49$ & 156020 & 160323 & 159956 & 160900 & 159445 & 160749 & 2.94 \\
\hline 50-54 & 137580 & 133768 & 134016 & 133358 & 133361 & 133360 & 3.16 \\
\hline $55-59$ & 107600 & 111412 & 111164 & & & 2.82 & \\
\hline
\end{tabular}


African Population Studies Vol 32, No. 3, Dec. 2018

\begin{tabular}{|c|c|c|c|c|c|c|c|}
\hline $60-64$ & 100150 & 95841 & 94740 & 94909 & 94779 & 90538 & 10.62 \\
\hline 65-69 & 69410 & 73719 & 74820 & 74651 & 73674 & 71036 & 2.29 \\
\hline 70-74 & 55500 & & & 53716 & & 52098 & 6.53 \\
\hline 75-79 & 30320 & & & 32104 & & 33722 & 10.09 \\
\hline
\end{tabular}

FEMALE POPULATION OF MALI, 1988

\begin{tabular}{|c|c|c|c|c|c|c|c|}
\hline AGE & REPORTED & $\begin{array}{l}\text { CARRIER } \\
\text { FARAG }\end{array}$ & K.KING & ARRIAGA & $\begin{array}{l}\text { UN MOVING } \\
\text { AVERAGE }\end{array}$ & $\begin{array}{l}\text { STRONG } \\
\text { MOVING } \\
\text { AVERAGE }\end{array}$ & \% ERROR \\
\hline $0-4$ & 830660 & & & 885297 & & 886667 & 6.32 \\
\hline $5-9$ & 805460 & & & 750823 & & 749453 & 7.47 \\
\hline 10-14 & 596040 & 623707 & 624377 & 621701 & 632284 & 618499 & 3.63 \\
\hline 15-19 & 545000 & 517333 & 516663 & 519339 & 520590 & 518846 & 5.04 \\
\hline $20-24$ & 415030 & 421158 & 423223 & 419953 & 428688 & 428888 & 3.23 \\
\hline $25-29$ & 359380 & 353252 & 351187 & 354457 & 356754 & 359930 & 0.15 \\
\hline 30-34 & 312780 & 309076 & 308112 & 307955 & 309211 & 299419 & 4.46 \\
\hline 35-39 & 251970 & 255674 & 256638 & 256795 & 256426 & 249919 & 0.82 \\
\hline $40-44$ & 210800 & 200150 & 201282 & 199517 & 203985 & 203876 & 3.40 \\
\hline $45-49$ & 151820 & 162470 & 161338 & 163103 & 162333 & 168207 & 9.74 \\
\hline $50-54$ & 146620 & 134644 & 134962 & 134190 & 134614 & 135916 & 7.88 \\
\hline 55-59 & 98580 & 110556 & 110238 & 111010 & 110718 & 110780 & 11.01 \\
\hline 60-64 & 103170 & 93956 & 92778 & 92884 & 92036 & 89555 & 15.20 \\
\hline 65-69 & 61660 & 70874 & 72052 & 71946 & 69913 & 69015 & 10.66 \\
\hline 70-74 & 53460 & & & 50586 & & 49220 & 8.61 \\
\hline 75-79 & 25930 & & & 28804 & & 30170 & 14.05 \\
\hline $80+$ & 29740 & & & & & & \\
\hline
\end{tabular}

Table 8. MALE POPULATION OF MALI, 2009

\begin{tabular}{|c|c|c|c|c|c|c|c|}
\hline AGE & REPORTED & $\begin{array}{l}\text { CARRIER } \\
\text { FARAG }\end{array}$ & K.KING & ARRIAGA & $\begin{array}{l}\text { UN MOVING } \\
\text { AVERAGE }\end{array}$ & $\begin{array}{l}\text { STRONG } \\
\text { MOVING } \\
\text { AVERAGE }\end{array}$ & \%ERROR \\
\hline
\end{tabular}


African Population Studies Vol 32, No. 3, Dec. 2018

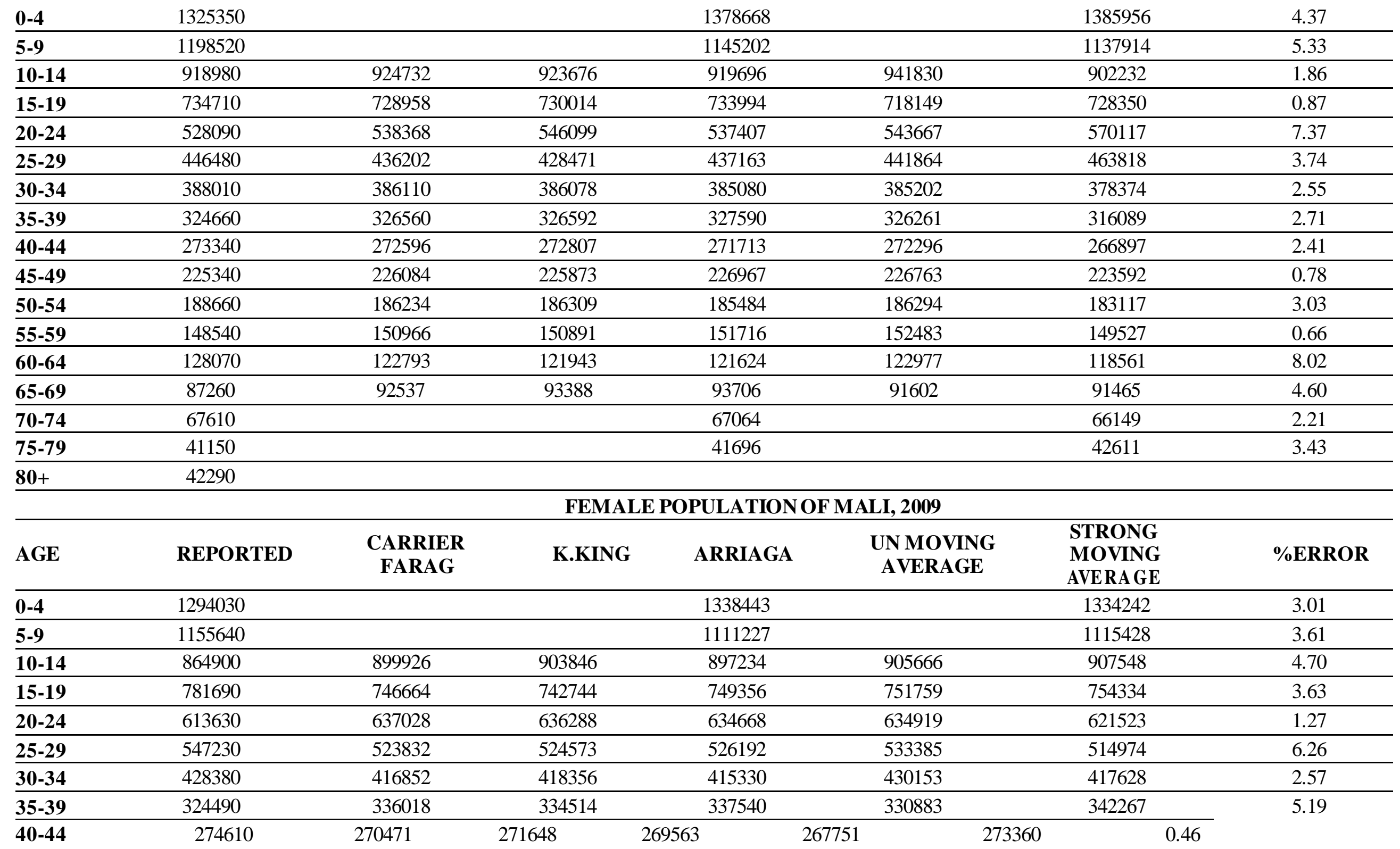


African Population Studies Vol 32, No. 3, Dec. 2018

\begin{tabular}{|c|c|c|c|c|c|c|c|}
\hline 45-49 & 215510 & 219649 & 218472 & 220557 & 223134 & 222667 & 3.21 \\
\hline 50-54 & 193710 & 181986 & 181855 & 181119 & 183558 & 179406 & 7.97 \\
\hline 55-59 & 133750 & 145474 & 145605 & 146341 & 144357 & 144405 & 7.38 \\
\hline $60-64$ & 122600 & 114456 & 114104 & 113480 & 113211 & 113213 & 8.29 \\
\hline 65-69 & 77520 & 85664 & 86016 & 86640 & 84944 & 86695 & 10.58 \\
\hline 70-74 & 66000 & & & 62298 & & 62405 & 5.76 \\
\hline $75-79$ & 36750 & & & 40453 & & 40345 & 8.91 \\
\hline
\end{tabular}

Table 9. MALE POPULATION OF SENEGAL, 1988

\begin{tabular}{|c|c|c|c|c|c|c|c|}
\hline AGE & REPORTED & $\begin{array}{l}\text { CARRIER } \\
\text { FARAG }\end{array}$ & K.KING & ARRIAGA & $\begin{array}{c}\text { UN MOVING } \\
\text { AVERAGE }\end{array}$ & $\begin{array}{l}\text { STRONG } \\
\text { MOVING } \\
\text { AVERAGE } \\
\end{array}$ & \%ERROR \\
\hline $0-4$ & 666100 & & & 678859 & & 672727 & 0.99 \\
\hline $5-9$ & 551090 & & & 538331 & & 544463 & 1.22 \\
\hline 10-14 & 406520 & 409508 & 413904 & 408538 & 416875 & 424167 & 4.16 \\
\hline 15-19 & 334430 & 331442 & 327046 & 332413 & 328304 & 343709 & 2.70 \\
\hline $20-24$ & 271180 & 285291 & 285404 & 284375 & 278699 & 279383 & 2.94 \\
\hline $25-29$ & 251140 & 237029 & 236916 & 237945 & 240659 & 230397 & 9.00 \\
\hline 30-34 & 188380 & 195671 & 195509 & 194784 & 198036 & 189002 & 0.33 \\
\hline 35-39 & 164670 & 157379 & 157541 & 158266 & 154181 & 155914 & 5.62 \\
\hline $40-44$ & 107280 & 119268 & 120748 & 118965 & 118849 & 125003 & 14.18 \\
\hline $45-49$ & 111300 & 99312 & 97832 & 99615 & 102580 & 103812 & 7.21 \\
\hline $50-54$ & 86710 & 91934 & 91520 & 91704 & 92375 & 87251 & 0.62 \\
\hline 55-59 & 83010 & 77786 & 78200 & 78016 & 78058 & 72464 & 14.55 \\
\hline $60-64$ & 59060 & 64640 & 63556 & 63667 & 63632 & 59738 & 1.13 \\
\hline 65-69 & 52960 & 47380 & 48464 & 48353 & 48884 & 45799 & 15.64 \\
\hline 70-74 & 29660 & & & 32596 & & 31662 & 6.32 \\
\hline $75-79$ & 19330 & & & 16394 & & 17328 & 11.55 \\
\hline
\end{tabular}


FEMALE POPULATION OF SENEGAL, 1988

\begin{tabular}{|c|c|c|c|c|c|c|c|}
\hline AGE & REPORTED & $\begin{array}{l}\text { CARR IER } \\
\text { FARAG }\end{array}$ & K.KING & ARRIAGA & $\begin{array}{l}\text { UN MOVING } \\
\text { AVERAGE }\end{array}$ & $\begin{array}{l}\text { STRONG } \\
\text { MOVING } \\
\text { AVERA GE }\end{array}$ & \%ERROR \\
\hline $0-4$ & 661530 & & & 684400 & & 677888 & 2.41 \\
\hline $5-9$ & 578140 & & & 555270 & & 561782 & 2.91 \\
\hline 10-14 & 415120 & 437317 & 441086 & 436105 & 440298 & 452293 & 8.22 \\
\hline $15-19$ & 387740 & 365543 & 361774 & 366755 & 368545 & 375897 & 3.15 \\
\hline $20-24$ & 308430 & 330327 & 328716 & 329189 & 325443 & 314167 & 1.83 \\
\hline $25-29$ & 296740 & 274843 & 276454 & 275981 & 276791 & 261384 & 13.53 \\
\hline 30-34 & 200010 & 215533 & 215831 & 214463 & 218858 & 212452 & 5.86 \\
\hline 35-39 & 184760 & 169237 & 168939 & 170308 & 168788 & 172714 & 6.97 \\
\hline $40-44$ & 115950 & 127309 & 128872 & 127042 & 129528 & 133981 & 13.46 \\
\hline $45-49$ & 114090 & 102731 & 101168 & 102998 & 104316 & 107953 & 5.68 \\
\hline 50-54 & 82450 & 90093 & 89734 & 89668 & 89750 & 87234 & 5.48 \\
\hline $55-59$ & 80690 & 73047 & 73406 & 73472 & 73847 & 70152 & 15.02 \\
\hline 60-64 & 51670 & 57933 & 57223 & 57073 & 57722 & 55616 & 7.10 \\
\hline 65-69 & 47740 & 41477 & 42188 & 42337 & 42664 & 41547 & 14.91 \\
\hline $70-74$ & 24420 & & & 28200 & & 27971 & 12.70 \\
\hline 75-79 & 18440 & & & 14660 & & 14889 & 23.85 \\
\hline
\end{tabular}

Table 10. MALE POPULATION OF SENEGAL, 2002

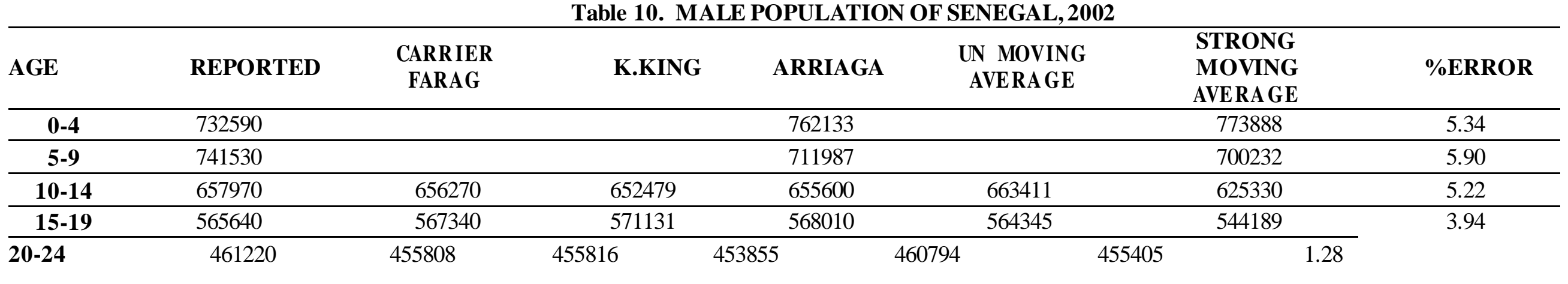


African Population Studies Vol 32, No. 3, Dec. 2018

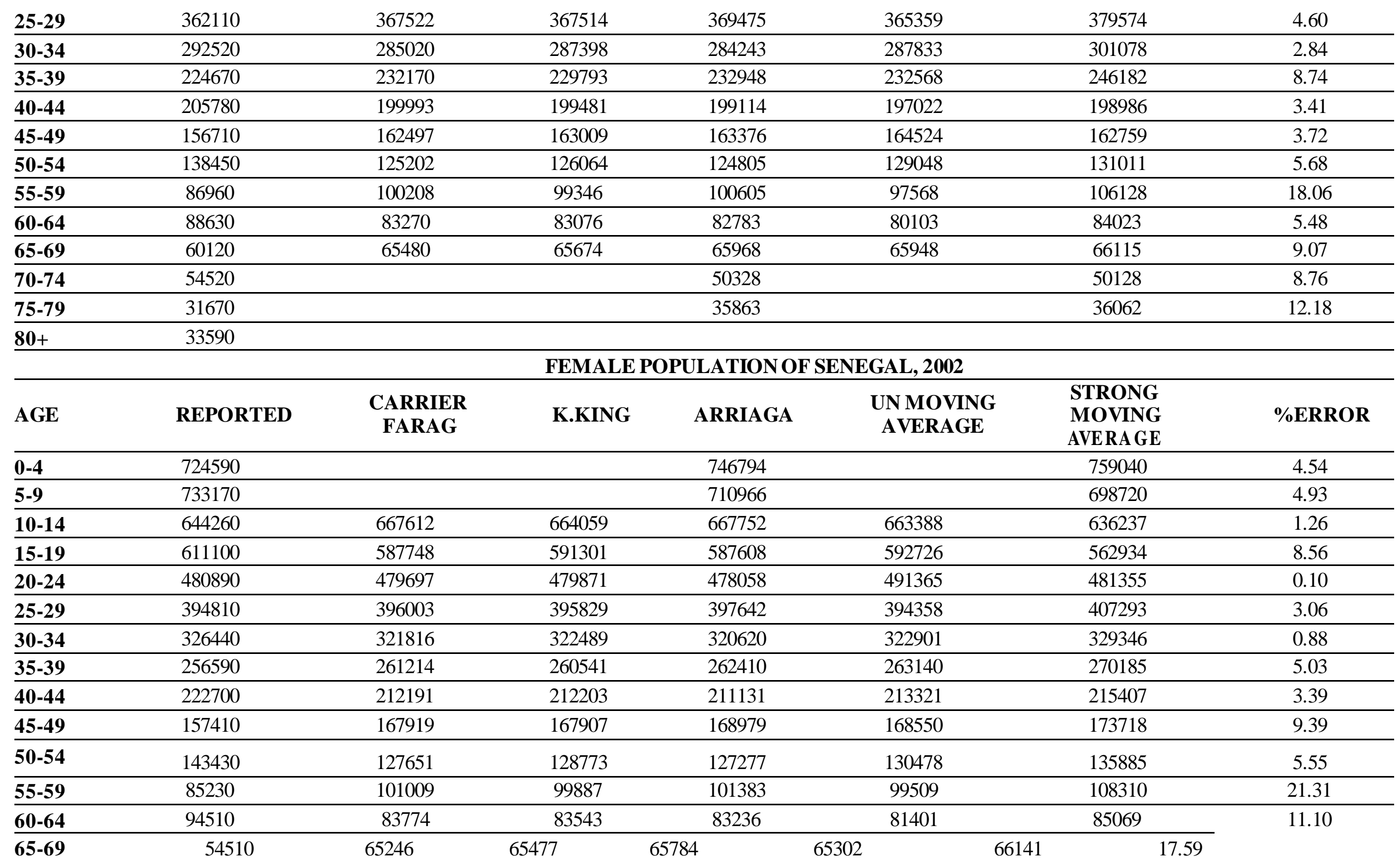


African Population Studies Vol 32, No. 3, Dec. 2018

70-74

75-79

80+
58210

25920

37170
49371

49562

34568

34759

17.90

25.43 


\section{Error detection}

Table I.I shows that there was a reduction in the level of inaccuracy (from 31.8 to 28.6) in Burkina Faso population data between years 1996 and 2006. Also, the average age ratio reduced for both sexes (Male 5.68 in 1996 to 4.80 in 2006; Female 8.74 in 1996 to 5.95 in 2006). The maximum positive and negative deviations in the male population in 1966 were in age group 60-64 (I 14.2\%) and 65-69 (13.4\%) respectively while in the female population it was noted in age groups 60-64 (20.7\%) and 65-69 $(19.4 \%)$ respectively. For the male population in 2006, the maximum positive and negative deviations were noted in 35-39 (5.9\%) and 55-59 (1 I.4\%); in the female population, it was observed that the maximum positive and negative deviations were in age groups 60-64 (11.5\%) and 55-59 (19\%) respectively.

Table 1.2 shows a reduction in the level of inaccuracy in Ghana male and female population data between years 2000 and 2010 (from 34.7 to 28.8). The average age ratio for the males ranged from 5.98 (in 2000) to 6.02 (in 2010), but there was no significant decline in the female population for between the years i.e. both approximated to 8 . In terms of the deviations, age group 60-64 and 55-59 have the maximum positive and negative deviations respectively in the male population in the year 2000 , but age groups 60-64 (13.3\%) and 65-59 (26.4\%) have the maximum positive and negative deviations respectively in 2010 . In the female population, it was observed that the maximum positive and negative deviations were in age groups 60-64 (24.5\%) and 6559 (16.6) in the year 2000. On the other hand, it was observed that age groups 60-64 (13.3\%) and 65-69 $(26.4 \%)$ have the maximum positive and negative deviations respectively in 2010 .

Table I.3 shows that there was a decline in the level of inaccuracy (from 38.3 to 27.9) in Cameroon population data between years 1987 and 2005 . Also, the average age ratio reduced for both sexes (males: 6.39 in 1987 to 3.81 in 2005 ; females 10.30 in 1987 to 6.75 in 2005). The maximum positive and negative deviations in the male population (in 1987) were in age groups 50-54 and 60-64 (12.8\% respectively) and 65-69 (24.1\%) respectively while in the female population it was noted in age groups 60-64 (25\%) and $65-69(24.1 \%)$ respectively. Again, for the male population in 2005 , the maximum positive and negative deviations were noted in 60-64 (6.8\%) and $55-59$ (13.9\%); in the female population, it was observed that the maximum positive and negative deviations were in age groups 60-64 (17.6\%) and 5559 (25.3\%) respectively.

According to Table 1.4, the age accuracy index ranged from 42.6 to 34.4 between Mali 1998 and
2009 population censuses. Besides, the average age ratio for the males ranged from 5.52 to 4.16 while it declined from II.II to 8.24. The maximum positive deviations in the 1998 male and female population were in age groups $60-64$ (13.2\% and $28.8 \%$ respectively. It was also observed in the age group $60-64$ in the male and female population $(8.6 \%$ and $16.1 \%$ respectively) in the year 2009 . With respect to the negative deviations, it was observed that the maximum negative deviation was $10.8 \%$ (in the age group 65-69) in years 1998 and 2009 in the male population while it ranged from $21.3 \%$ to $17.8 \%$ in the same age groups in the female population in 2009.

According to Table I.5, that there was a decline in the level of inaccuracy (from 44.8 to 39.5) in Senegal population data between years 1988 and 2002. Also, the average age ratio reduced for both sexes (males: I 0.29 in I 988 to 8.1 I in 2002; females I4.79 in 1988 to 11.80 in 2002). The maximum positive deviations in the Senegal 1988 male and female population were in age groups $65-69$ (19.4\% and $25.5 \%$ respectively). It was also observed in the age group 60-64 in the male and female population ( $20.5 \%$ and $35.3 \%$ respectively) in the year 2002 . With respect to the negative deviations, it was observed that the maximum negative deviation was $22.3 \%$ and $22.4 \%$ (in the age group $40-44$ ) in the male and female population respectively in the year 1998. It was $23.4 \%$ (55-59) in the male population and $28.6 \%(65-69)$ in the female population in 2002.

\section{Age smoothing \\ Male and female population of Burkina Faso, 1996 and 2006}

Table I. shows that in 1996, males and females in the younger age groups were less under enumerated than older age groups. Males belonging to age group 60-64 (11\%) where largely over-enumerated. Females belonging to age group 75-79 (I 7\%) where largely over-enumerated while those in the group 3539 (1\%) were the most under-enumerated. Table 2. shows that in 2006, males belonging to age group 5-9 and 75-79 where over-enumerated while those in the group 35-39 (1\%) were the most under-enumerated. Females belonging to age group 75-79 (1 I\%) where largely over-enumerated.

\section{Male and female population, Ghana 2000 and 2010}

Table 3 shows that in 2000 , males and females in the younger age groups were less under-enumerated than older age groups. Males belonging to age group $55-59$ (18\%) where largely over-enumerated while those in the group 25-29 (I\%) were the most underenumerated group. Females belonging to age group 
55-59 where largely over-enumerated while those in the group 10-14 were under-enumerated. Table 4 shows that over-enumeration was largely present in both males and females in older age groups. Those male populations in the groups 65-69 (22\%) and 6064 (1\%) were the most over-enumerated and underenumerated groups respectively. Females belonging to age groups 65-69 (23\%) and 60-64 (I\%) where largely over-enumerated and under-enumerated respectively.

Male and female population, Cameroon, I 987 and 2005

Table 5 shows that in 1987, males and females in the younger age groups were less under-enumerated than older age groups. Males belonging to age group 75-79 (I7\%) where largely over-enumerated while those in the group 40-44 (I\%) were underenumerated. Females belonging to age group 75-79 $(25 \%)$ where largely over-enumerated while those in the group 45-49 (I\%) were the most underenumerated group. Table 6 shows that overenumeration was largely present in both males and females in older age groups. Those male populations in the groups 75-79 $(13 \%)$ and $45-49(1 \%)$ were the most over-enumerated and under-enumerated groups respectively. Females belonging to age groups 55-59 (20\%) and 60-64 (4\%) where largely overenumerated and under-enumerated respectively.

Male and female population, Mali, 1988 and 2009 Table 7 shows that in 1988, males and females in the younger age groups were less under-enumerated than older age groups. Males belonging to age group 60-64 (11\%) where largely over-enumerated but those in 30-34 (1\%) were under-enumerated. Females belonging to age group 75-79 (I4\%) where largely over-enumerated while those in the group 35 39 (I\%) were under-enumerated. Table 8 shows that in 2006, males belonging to age group 20-24 $(7 \%)$ and $15-19$ (1\%) were the most overenumerated and under-enumerated respectively. Females belonging to age group 65-69 (1 I \%) where largely over-enumerated while those in $40-44$ (0.5\%) were under-enumerated.

Male and female population of Senegal, I 988 and 2002

Table 9 shows that in 1988, males in the younger age groups were less under-enumerated than older age groups. Males belonging to age group 65-69 (16\%) where largely over-enumerated but those in 0-4 ( $1 \%)$ were under-enumerated. Females belonging to age group $75-79$ (24\%) where largely overenumerated. Table 8 shows that in 2002, males belonging to age group $75-79$ (I8\%) were the most over-enumerated. Females belonging to age group 65-69 (25\%) where largely over-enumerated while those in $30-34(0.5 \%)$ were the most underenumerated.

\section{Discussion and policy implications}

Having applied the smoothing technique, to the two census periods observed in this study, an upward pattern of over-enumeration ranging between 10 $25 \%$ was noticed for both sexes in the older ages. Besides, women were more likely to be overestimated than men in virtually all the countries save the male and female census data of Cameroon in 2005 and Senegal in 2002, in which both males and females were equally over-enumerated. Studies have established that age misreporting results from imbalances in the age-sex population coverage within the technical and political contexts and similarly from resulting contents errors associated with the enumerators' error which is a more plausible reason for the obvious age misreporting (Adebowale et al., 2012; Gibril, 1979; Mba, 2004). In addition, misreporting of true ages, either irrespective of the sex age, particularly at older ages, is typical of Africa as earlier reported (Siegel, 2004).

On the other hand, an unchanged proportion of under-enumeration was a usual pattern among the females i.e. (I\%) under-enumeration among those aged 35-39 in Burkina-Faso, in Ghana, ages 10-14, 45-49 in Cameroun and 35-39 in Mali and the least $(0.5 \%)$ in Senegal. Nevertheless, a sharp spike was noticed for males in ages 5-9 in the earlier census (1996) but not observed in the latter census (2006) in Burkina-Faso, also in Mali, about $7 \%$ overenumeration was observed for the male adolescents (20-24years). This unsustained distortion observed especially among the male population might be indicative of increasing literacy and knowledgeability of mothers in knowing their wards' birthdays in their early years of life, and maybe the presence of other more enlightened older children in helping with age reporting and sometimes, perhaps children's rapid growth as a result of improved nutrient uptake may be pivotal in misleading the enumerators to assume and report wrong ages. In the same vein, past observations in these countries (Ewbank, 1981; Gendreau \& Nadot, 1967; Hertrich \& Lardoux, 2014; Mba, 2014) showed that adolescents often times possess a lot of attitudinal and behavioral challengeswhich may not be un-connected with peer pressure, indecisions and preferences- that gives rise to inaccuracies in age reporting.

The highly prevalent habitual age misreporting in African countries could be alluded to lack of birth certificate, recall lapse, deliberate age shifting, digit preference, age heaping which render any 
subsequent analyses inaccurate and unreliable (Makannah, 1990; Mba, 2003, 20I4; Gilles Pison \& Ohadike, 2006; Siegel, 2004; Williams, 20I4); unfortunately this trend is not divorceable from administrative benefits derivable from age misreporting. In light of these findings, it is crystal clear on reasons planning and programmatic interventions especially based on census data have continued to suffer huge setbacks (Adebowale et al., 2012; Mba, 2004, 2006; Randall \& Coast, 20 I6; UN, 1967) amidst increasing literacy rate, high global awareness of quality census exercise as well as increased external census funding among others in sub-Saharan African countries.

\section{Conclusion}

This study found that the strong smoothing method, among other smoothing techniques was effective in unmasking and adjusting the error-ridden census data of all the selected West African countries. Hence, it is recommended that alongside implementing a great deal of data quality assurance operations in enumeration exercises, the strong smoothing technique should be adopted as a better way of unearthing and adjusting census data in West Africa and perhaps in Africa compared to the errorconcealing tendency of the light smoothing techniques.

\section{References}

Adebowale, A. S., Fagbamigbe, A. F., \& Bello, A. S. $(20 \mid 2)$. Refined age distribution and demographic parameters estimation in nigeria: An indirect approach. Journal of Statistics and Management Systems, I5(I), 29-48.

Arriaga, E., Johnson, P., \& Jamison, E. (1994). Population analysis with microcomputers: presentation of techniques: Bureau of the Census. United States, I.

Axinn, W. G., Pearce, L. D., \& Ghimire, D. (1999). Innovations in life history calendar applications. Social Science Research, 28(3), 243-264.

Carrier, N. H., \& Farrag, A. (1959). The reduction of errors in census populations for statistically underdeveloped countries. Population Studies, I2(3), 240-285.

Chevan, A., \& Sutherland, M. (2009). Revisiting Das Gupta: Refinement and extension of standardization and decomposition. Demography, 46(3), 429-449.

Coale, A. J., \& Kisker, E. E. (1986). Mortality crossovers: Reality or bad data? Population Studies, 40(3), 389-40I.

Ewbank, D. C. (|98I). Age misreporting and ageselective underenumeration: sources patterns and consequences for demographic analysis.
Gendreau, F., \& Nadot, R. (1967). Afrique Noire, Madagascar, Comores, Démographie Comparée. Structures par âge, actuelle et future. DGRST, Paris, 9l.

Gibril, M. (1979). Evaluating census response errors: a case study for the Gambia. Development Centre Studies.

Helleringer, S., Pison, G., Masquelier, B., Kanté, A. M., Douillot, L., Duthé, G., . . . Delaunay, V. (20|4). Improving the quality of adult mortality data collected in demographic surveys: validation study of a new siblings' survival questionnaire in Niakhar, Senegal. PLoS medicine, II(5), el 001652.

Hertrich, V., \& Lardoux, S. (20|4). Âge à l'entrée en union des femmes en Afrique. Les données des enquêtes et des recensements sont-elles comparables? Population, 69(3), 399-43I.

Hobbs, F. (2004). Age and sex composition. The methods and materials of demography, 2, I25173.

Krótki, K. J. (1978). Developments in dual system estimation of population size and growth: University of Alberta.

Makannah, T. (1990). An evaluation of the age-sex data of recent African censuses. Paper presented at the sixth session of the Joint Conference of African Planners, Statisticians and Demographers, United Nations Economic Commission for Africa, Addis Ababa, Ethiopia.

Masquelier, B., Ndiaye, C. T., Pison, G., Dieme, N. B., Diouf, I., Helleringer, S., . . . Delaunay, V. (20|6). Evaluation des estimations indirectes de mortalité dans trois observatoires de population au Sénégal. African Population Studies, 30(I), 2227-224I.

Mba, C. J. (2003). Assessing the reliability of the 1986 and 1996 Lesotho census data. Journal of Social Development in Africa, I 8(I).

Mba, C. J. (2004). Challenges of population census enumeration in Africa: an illustration with the agesex data of the Gambia. Institute of African Studies Research Review, 20(I), 9-19.

Mba, C. J. (2006). Reliability of Age-Sex Data in Demographic Analysis: An assessment of Nigeria's empirical evidence, 1990-2003. Ibadan Journal Of The Social Sciences, 4(2), 89-102.

Mba, C. J. (20|4). 2 Examining the Accuracy of AgeSex Data. Continuity and Change in Sub-Saharan African Demography, 12.

Pison, G., Hill, K., Cohen, B., \& Foote, K. (1995). Population dynamics of Senegal, Washington, Report of the Committee on Population of the National Academy of Science: National Academy Press. 
Pison, G., \& Ohadike, P. (2006). Errors and manipulations in age assessment. Human Clocks. The Bio-Cultural Meaning of Age, Peter Lang, Berne, 3| 3-336.

Randall, S., \& Coast, E. (20|6). The quality of demographic data on older Africans. Demographic Research, 34, I 43.

Raymer, J., \& Rogers, A. (2007). Using age and spatial flow structures in the indirect estimation of migration streams. Demography, 44(2), 199-223.

Sembajwe, I. S. L. (1990). The evaluation of the vital registration system in Lesotho: The case of Mantsebo: Demography Unit, Dept. of Statistics, National University of Lesotho.

Siegel, J. S., and Swamson D.A, eds. . (2004). The Methods and Materials of Demography.2nd ed. Washington, DC: Census Bureau.

Spoorenberg, T. (2007). Quality of age reporting: extension and application of the modified Whipple's index. Population, 62(4), 729-74I.
Udjo, E. O. (2005). An examination of recent census and survey data on mortality within the context of HIV/AIDS. The Demography of South Africa.

UN. (1967). Department of Economic and Social Affairs Manual IV: Methods of Estimating Basic Demographic Measures from Incomplete Data. Population Studies No. 42. New York: United Nations.

UN. (I 986 ). Demographic Yearbook. New York: United Nations.

UN. (20 I6). United Nations Statistics Division. 2020 World Population and Housing Census Programme Department of Economic and Social Affairs [UNDESA].

UN. ( 1955). Manual II Methods of appraisal of quality of basic data for population estimates $\mathrm{New}$ York, United Nations

Williams, A. O. (20|4). Assessment of the completeness of births and deaths registration in an urban Nigerian community. Etude de la Population Africaine, 27(2), 263. 